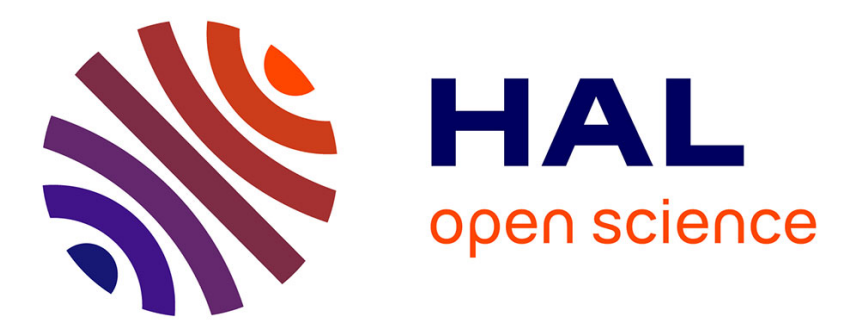

\title{
Interpretation of the reduced density gradient
}

Roberto A. Boto, Julia Contreras-García, Julien Tierny, Jean-Philip Piquemal

\section{To cite this version:}

Roberto A. Boto, Julia Contreras-García, Julien Tierny, Jean-Philip Piquemal. Interpretation of the reduced density gradient. Molecular Physics, 2015, pp.1-9. 10.1080/00268976.2015.1123777 . hal01253888

\section{HAL Id: hal-01253888 \\ https://hal.science/hal-01253888}

Submitted on 11 Jan 2016

HAL is a multi-disciplinary open access archive for the deposit and dissemination of scientific research documents, whether they are published or not. The documents may come from teaching and research institutions in France or abroad, or from public or private research centers.
L'archive ouverte pluridisciplinaire $\mathbf{H A L}$, est destinée au dépôt et à la diffusion de documents scientifiques de niveau recherche, publiés ou non, émanant des établissements d'enseignement et de recherche français ou étrangers, des laboratoires publics ou privés. 


\title{
Interpretation of the reduced density gradient
}

\author{
Roberto A. Boto $^{a, b *}$, Julia Contreras-García ${ }^{b, c}$, Julien Tierny ${ }^{d, e}$ and Jean-Philip \\ Piquemal ${ }^{b, c}$ \\ ${ }^{a}$ Sorbonne Universités, UPMC Univ Paris 06, ICS, Paris, France \\ ${ }^{b}$ Sorbonne Universités, UPMC Univ Paris 06, UMR 7616, Laboratoire de Chimie \\ Théorique, Paris, France \\ ${ }^{c}$ CNRS, UMR 7616, Laboratoire de Chimie Théorique, Paris, France \\ ${ }^{d}$ Sorbonne Universités, UPMC Univ Paris 06, UMR 7606, LIP6, Paris, France \\ ${ }^{e}$ CNRS, UMR 7606, LIP6, Paris, France
}

\begin{abstract}
An interpretation of the reduced density gradient in terms of the bosonic kinetic energy density is presented. Contrary to other bonding indicators based on the kinetic energy density such as the localised orbital locator (LOL) or the electron localization function (ELF), the reduced density gradient is not only able to identify covalent bondings, but also ionic and non-covalent interactions. This study reveals that the critical points of the reduced density gradient are closely connected with those of LOL and with the roots of the one-electron potential (OEP).
\end{abstract}

Keywords: kinetic-energy density, reduced density gradient, ELF, LOL, OEP

\section{Introduction}

Recently some of us have developed the non-covalent interactions (NCI) method to detect NCI in real space [1]. This method enables the identification of NCI as isosurfaces of the reduced density gradient $s: \mathbb{R}^{3} \rightarrow \mathbb{R}$,

$$
s(\mathbf{r})=\frac{|\nabla \rho(\mathbf{r})|}{2\left(3 \pi^{2}\right)^{1 / 3} \rho(\mathbf{r})^{4 / 3}} .
$$

These isosurfaces expand over large regions of space containing interacting atoms. This approach recovers a more intuitive picture of van der Waals interactions, hydrogen bonds and steric repulsions than other local pairwise approaches such as atoms in molecules (AIM) theory, or ELF. The application of the reduced density gradient to visualise interacting regions, was motivated by the deviations from the exponential behaviour observed when $s$ is plotted versus $\rho$ for interacting systems

Nevertheless, the original formulation of the reduced density gradient was not introduced within chemical bonding theories, but within the generalised gradient contribution to the generalised gradient approximation (GGA) exchange energy, $E_{x}^{G G A}$, from the density functional theory (DFT):

$$
E_{x}^{G G A}[\rho \uparrow(\mathbf{r}), \rho \downarrow(\mathbf{r})]=\int_{\mathbb{R}^{3}} \rho(\mathbf{r}) \epsilon_{\mathbf{x}}^{\text {unif }}(\rho(\mathbf{r})) \mathbf{F}_{\mathbf{x}}(\mathbf{s}(\mathbf{r})) \mathbf{d r} \mathbf{r}
$$

\footnotetext{
${ }^{*}$ Corresponding author. Email: alboto@lct.jussieu.fr
} 
where $\rho \uparrow(\mathbf{r})$ and $\rho \downarrow(\mathbf{r})$ are the spin-up and spin-down electron densities respectively, $\rho(\mathbf{r})=\rho \uparrow(\mathbf{r})+\rho \downarrow(\mathbf{r})$ is the total electron density, $\epsilon_{\mathbf{x}}^{\text {unif }}(\rho(\mathbf{r}))=$ $-(\mathbf{3} / \mathbf{4})(\mathbf{3} / \pi)^{\mathbf{1} / \mathbf{3}}(\rho(\mathrm{r}))^{\mathbf{1} / \mathbf{3}}$ is the exchange energy per electron of the uniform electron gas, and $\mathbf{F}_{\mathbf{x}}(\mathbf{s}(\mathbf{r}))$ is the enhancement factor whose functional form defines the exchange contribution to the energy within the GGA approximation [2]. Since the reduced density gradient is one of the ingredients to correct the uniform electron gas model, a lot of work has been devoted to analyse its properties [3, 4, 5]. As many other dimensionless parameters introduced in the context of semilocal meta-GGAs [6], the reduced density gradient is not a monotonic function in atomic systems, that is, it exhibits a pattern of maxima and minima. This behaviour has been used to identify atomic shells. Using this property, Zupan et al. proposed an analysis of local or semilocal functionals, based on the distribution of the values of the reduced density gradient and the Seitz radius in atoms, solids and surfaces [7, 8]. In subsequent work, del Campo et al. , applied the same procedure to set the basic properties of the reduced density gradient in atoms [9].

Recently Finzel showed that several functions derived from the kinetic energy density, and developed as energetic corrections in density functionals, are able to reproduce atomic shell structure to different extents, and therefore, are promising chemical bonding descriptors [10]. This is the case of ELF, LOL, the Becke-Johnson potential and many other dimensionless parameters [11, 12, 13]. The connection of all these functionals with the kinetic energy density is the key to understand their success as bonding descriptors in real space; any sort of covalent interaction is followed by a decrease in the interatomic kinetic energy, therefore, any function able to map this variations should be a good candidate as a real space bonding indicator. Conversely, it has been demonstrated that such descriptors only shed light on the covalent bonding description, and are not able to identify NCI [14, 15]. The NCI method has come up to fill the gap between both interaction types. Although mainly applied to NCI, it can also be used to detect covalent bonds and ionic interactions [1, 16]. Thus, the applicability of the reduced density gradient ranges from atomic shell structure to NCI. In spite of providing an intuitive image of chemical bonding, the NCI index needs to be developed in two main aspects. On the one hand, there is not a clear physical meaning of $s(\mathbf{r})$. In DFT, $s(\mathbf{r})$ is understood as a measure of the inhomogeneity of the system, and therefore, as a correction to the uniform electron gas. Regions where $s(\mathbf{r})$ takes large and small values were analysed, independently of the oscillation within such regions. Bohórquez et al. proposed an alternative definition as a relative measure of the local momentum [17]. On the other hand, the lack of physical meaning of $s(\mathbf{r})$ hampers a direct connection with energetics. Contreras et al. defined a strategy to define NCI regions and to integrate molecular properties within them [18]. Following this approach, a close connection between properties integrated in such regions and the stabilization energy in systems governed by dispersive interactions has been shown [19, 20]. However, the origin of such correlation is still unclear.

In this work we discuss the chemical content of the reduced density gradient, and we show that beyond a dimensionless DFT parameter, $s(\mathbf{r})$ may be included in the family of chemical descriptors based on the kinetic energy density such as LOL or ELF. Subsequently, we rationalise the ability of such functions to reveal NCI.

\section{Theory}

The Pauli kinetic energy density $t_{p}(\mathbf{r})$ is defined as the excess of kinetic energy compared with a system of bosons of the same density [21]. For a single determinantal wavefunction, it may be expressed as the difference between the positive 
kinetic energy density $\tau(\mathbf{r})=1 / 2 \sum_{i} \nabla \phi_{i}^{*}(\mathbf{r}) \nabla \phi_{i}(\mathbf{r})$ and the von Weizsäcker term $\tau_{w}(\mathbf{r})=1 / 8(\nabla \rho(\mathbf{r}))^{2} / \rho(\mathbf{r})$. Here $\rho(\mathbf{r})=\sum_{i} \phi_{i}^{*} \phi_{i}$ is the electron density and $\phi_{i}$ are the spinorbitals. Scaling this kinetic energy density by the Thomas-Fermi term $\tau_{T F}(\mathbf{r})=C_{F} \rho(\mathbf{r})^{5 / 3}$ where $C_{F}=3 / 10\left(3 \pi^{2}\right)^{2 / 3}$, we obtain the ELF kernel, $\chi(\mathbf{r})$

$$
\chi(\mathbf{r})=\frac{t_{p}(\mathbf{r})}{\tau_{T F}(\mathbf{r})}=\frac{\tau(\mathbf{r})-\tau_{w}(\mathbf{r})}{\tau_{T F}(\mathbf{r})} .
$$

Becke and Edgecombe introduced $t_{p}(\mathbf{r})$ as an indicator of the probability of finding and electron in the vicinity of a same spin reference electron. The smaller the probability density, the higher the probability of finding an opposite spin pair of electrons, and therefore, the Pauli principle is recovered [11]. Although all the chemical meaning of ELF is contained in $t_{p}(\mathbf{r})$ as the only measure of electron localisation [22], it is known that $t_{p}(\mathbf{r})$ is not able to reveal chemical structure features (see Supporting Information, Figure S1). As explained by Schmider et al. [23] the origin of this may be found in the scaling relation between the kinetic energy $\tau(\mathbf{r})$ and the density; $\tau(\mathbf{r})$ is completely dominated by the core regions. To circumvent this problem, Becke and Edgecombe chose $\tau_{T F}(\mathbf{r})$ as reference. Within the local quantum theory framework, Bohórquez et al. chose the electron density as reference [24]. They analysed the local values of all the functions involved in $t_{p}(\mathbf{r})$, $t_{p}(\mathbf{r}) / \rho(\mathbf{r}), \tau(\mathbf{r}) / \rho(\mathbf{r})$ and $\tau_{w}(\mathbf{r}) / \rho(\mathbf{r})$. They have not only shown, that $t_{p}(\mathbf{r}) / \rho(\mathbf{r})$ provides a better depiction of electron localisation than $t_{p}(\mathbf{r})$, but also $\tau(\mathbf{r}) / \rho(\mathbf{r})$ and $\tau_{w}(\mathbf{r}) / \rho(\mathbf{r})$ are useful chemical bonding descriptors. Conversely, the chemical content of $\tau(\mathbf{r})$, was already used by Schmider and Becke to define the LOL descriptor. LOL is defined from the dimensionless variable $t(\mathbf{r})$,

$$
t(\mathbf{r})=\frac{\tau_{T F}(\mathbf{r})}{\tau(\mathbf{r})} .
$$

The function $t(\mathbf{r})$ is bounded by zero from below, but has no an upper boundary,

$$
0 \leq t(\mathbf{r})<\infty
$$

To circumvent this problem, they proposed to map $t(\mathbf{r})$ onto the range $[0,1]$. They referred to this map as $\nu$,

$$
\nu(\mathbf{r})=\frac{t(\mathbf{r})}{1+t(\mathbf{r})}=\frac{1}{1+\frac{\tau(\mathbf{r})}{\tau_{T F}(\mathbf{r})}} .
$$

LOL refers both to $t(\mathbf{r})$ and to its bounded counterpart $\nu(\mathbf{r})$. It may be noticed, that the inverse of $t(\mathbf{r})$ is the first right term of Equation 1. In what follows, we shall refer to $t_{L O L}(\mathbf{r})=\tau(\mathbf{r}) / \tau_{T F}(\mathbf{r})$ as the LOL kernel. The properties of this dimensionless ratio were already discussed by Finzel [10]. Contrary to $\tau / \rho$, $t_{L O L}(\mathbf{r})$ is not a bounded function. At the positions of the stationary points of localised orbitals, $t_{L O L}(\mathbf{r})$ is driven to small values $(\nu \rightarrow 1)$. In regions dominated by the overlap of localised orbitals, $t_{L O L}(\mathbf{r})$ attains large values $(\nu \rightarrow 0)$. Thus, the chemical content of LOL is similar to that of ELF.

The term $\tau_{w}(\mathbf{r})$ in Equation 1, is the kinetic energy density in the absence of the Pauli principle, hence it accounts for the bosonic character of the system. $\tau_{w}(\mathbf{r})$ 
constitutes a rigorous lower bound for $\tau(\mathbf{r})$, it exhibits the same limiting behaviour at $\mathbf{r}=0$ and $\mathbf{r} \rightarrow \infty$ and it is exact for any system described by a single spatial orbital. In many electron systems, it also approaches $\tau(\mathbf{r})$ for those systems that are well described by strongly localised orbitals in separate regions. Additionally, $\tau_{w}(\mathbf{r})$ is the kinetic energy density of the marginal probability amplitude introduced by Hunter some time ago [25]. $\tau_{w}(\mathbf{r})$ may be thereby understood as a measure of the single particle character of the system. Because a localised electron pair behaves as a single particle, namely as a boson, its kinetic energy density is given by $\tau_{w}(\mathbf{r})$. This was already noticed by Bohórquez et al. who proposed a partition of molecular space based on the local behaviour of $\tau_{w}(\mathbf{r}) / \rho(\mathbf{r})$, valid for every pair of atoms connected through a bond critical point (BCP) 24]. They also introduced the localised electron detector (LED) $(\tilde{P}(\mathbf{r}))$ defined as the local momentum associated with $\tau_{w}(\mathbf{r}) / \rho(\mathbf{r})$,

$$
\tilde{P}(\mathbf{r})=-\frac{\nabla \rho(\mathbf{r})}{2 \rho(\mathbf{r})}
$$

In line with their work, they interpreted $s(\mathbf{r})$ as scaled momentum, where the scaling function is the Thomas-Fermi momentum $p_{T F}(\mathbf{r})=\left(3 \pi^{2} \rho(\mathbf{r})\right)^{1 / 3}[17$,

$$
s(\mathbf{r})=\frac{|\tilde{P}(\mathbf{r})|}{p_{T F}(\mathbf{r})} .
$$

\section{Results}

Similar to $t_{L O L}(\mathbf{r})$, we may rescale $\tau_{w}(\mathbf{r})$ by $\tau_{T F}(\mathbf{r})$ defining the dimensionless variable $t_{\text {bose }}(\mathbf{r})$ as,

$$
t_{\text {bose }}(\mathbf{r})=\frac{\tau_{w}(\mathbf{r})}{\tau_{T F}(\mathbf{r})}
$$

$t_{\text {bose }}(\mathbf{r})$ carries the same chemical information as $\tau_{w}(\mathbf{r})$; they account for the single particle character of the system. It is easy to notice that $\chi(\mathbf{r})$ is nothing but the difference between $t_{L O L}(\mathbf{r})$ and $t_{\text {bose }}(\mathbf{r})$. Similar to $\tau_{w}(\mathbf{r})$ and $\tau(\mathbf{r}), t_{\text {bose }}(\mathbf{r})$ is a lower bound to $t_{L O L}(\mathbf{r})$, and they approach each other in regions well described by a single orbital, as it occurs where there is electron pair localisation [21]. We may establish a parallelism between both functions: if $\mathrm{t}_{L O L}$ is understood as an indicator of positions where localised orbitals attain their stationary points, $\mathrm{t}_{\text {bose }}$ may be understood as a locator of the stationary points of the electron density. As explained by Savin [26], at regions where localised orbitals attain their maxima, $\nabla \rho(\mathbf{r})$ is expected to be close to 0 , and both functions $t_{L O L}$ and $t_{b o s e}$ are driven to small values. Thus, one requirement though not sufficient for electron localisation is small values of $\nabla \rho(\mathbf{r})$, and therefore, of $\tau_{w}(\mathbf{r})$ as well as $\mathrm{t}_{\text {bose }}$. Note that the opposite is not true; not all regions of low values of $\nabla \rho(\mathbf{r})$ involve maxima of localised orbitals. In this regard $t_{\text {bose }}(\mathbf{r})$ contains more chemical information than $t_{L O L}(\mathbf{r})$; it is not only able to provide regions of electron pair localisation in particular, but regions of electron localisation in general. This interpretation is also valid for any function derived from $\tau_{w}(\mathbf{r})$, such as $\tau_{w}(\mathbf{r}) / \rho(\mathbf{r})$ or LED. As noted by Bohórquez et al. [17] both $\tau_{w}(\mathbf{r}) / \rho(\mathbf{r})$ and LED are bounded by physical limits, whereas $t_{\text {bose }}(\mathbf{r})$ is an unbounded variable. For the shake of comparison with LOL, we define 


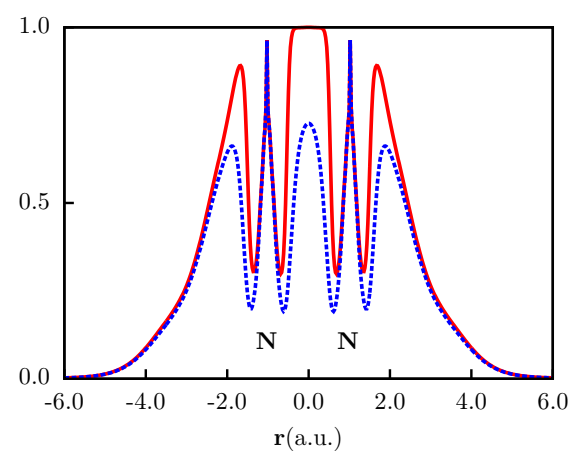

Figure 1. $\beta$ (solid red line) and LOL (dashed blue line) along internuclear axis for $\mathrm{N}_{2}$. The zero was set at the BCP.

$\beta(\mathbf{r})=1 / 1+t_{\text {bose }}(\mathbf{r})$, as an upper limit to $\nu(\mathbf{r})$ (Figure 1). It may be seen that the chemical picture obtained by the two functions is the same.

It may be straightforwardly shown that $s(\mathbf{r})$ is the kernel of $t_{\text {bose }}(\mathbf{r})$,

$$
\begin{aligned}
s(\mathbf{r}) & =\left(\frac{3}{10}\right)^{1 / 2} \frac{1}{2\left(C_{F}\right)^{1 / 2}} \frac{|\nabla \rho(\mathbf{r})|}{\rho(\mathbf{r})^{4 / 3}}, \\
t_{\text {bose }}(\mathbf{r}) & =\frac{\tau_{w}(\mathbf{r})}{\tau_{T F}(\mathbf{r})}=\left(\frac{5}{3}\right) s(\mathbf{r})^{2} .
\end{aligned}
$$

We may notice that its critical points $(\mathrm{CPs})$ match with those of $t_{\text {bose }}(\mathbf{r})$,

$$
\nabla t_{\text {bose }}(\mathbf{r})=\frac{10}{3} s(\mathbf{r}) \nabla s(\mathbf{r})
$$

Since $s(\mathbf{r})$ is positive semidefinite, at CPs the signs of their Laplacians are the same,

$$
\nabla^{2} t_{\text {bose }}(\mathbf{r})=\frac{10}{3}\left(\nabla s(\mathbf{r}) \nabla s(\mathbf{r})+s(\mathbf{r}) \nabla^{2} s(\mathbf{r})\right)
$$

at $\operatorname{CPs} \nabla t_{\text {bose }}\left(\mathbf{r}_{\mathbf{c p}}\right)=\nabla s\left(\mathbf{r}_{\mathbf{c p}}\right)=0$, and

$$
\nabla^{2} t_{\text {bose }}\left(\mathbf{r}_{\mathbf{c p}}\right)=\frac{10}{3}\left(s\left(\mathbf{r}_{\mathbf{c p}}\right) \nabla^{2} s\left(\mathbf{r}_{\mathbf{c p}}\right)\right) .
$$

Thus, the CPs of $s(\mathbf{r})$ and $t_{\text {bose }}(\mathbf{r})$ are identical both in location and in nature. Because of the presence of the gradient of $\rho$ in the numerator, and their positive semidefinite condition, at every $\mathrm{CP}$ of $\rho(\mathbf{r})$ both functions cancel and have a minimum. This result may be clearly shown, if we develop the explicit expression for $\nabla t_{\text {bose }}(\mathbf{r})$

$$
\nabla t_{\text {bose }}(\mathbf{r})=\frac{1}{4 C_{F}} \frac{\nabla \rho(\mathbf{r})}{\rho(\mathbf{r})^{5 / 3}}\left[\frac{\nabla^{2} \rho(\mathbf{r})}{\rho(\mathbf{r})}-\frac{4}{3} \frac{(\nabla \rho(\mathbf{r}))^{2}}{\rho(\mathbf{r})^{2}}\right]
$$

From this expression we may differentiate two situations where $\nabla t_{\text {bose }}(\mathbf{r})$ is can- 
celled:

(1) AIM-CPs: CPs of $\rho(\mathbf{r})$, for which $\nabla \rho(\mathbf{r})=0$.

(2) Non-AIM-CPs: Points where:

$$
\frac{\nabla^{2} \rho(\mathbf{r})}{\rho(\mathbf{r})}-\frac{4}{3} \frac{(\nabla \rho(\mathbf{r}))^{2}}{\rho^{2}(\mathbf{r})}=0 .
$$

At AIM-CPs $\nabla^{2} t_{\text {bose }}(\mathbf{r})$ takes the following form:

$$
\nabla^{2} t_{\text {bose }}(\mathbf{r})=\frac{1}{4 C_{F}} \frac{\left(\nabla^{2} \rho(\mathbf{r})\right)^{2}}{\rho(\mathbf{r})^{8 / 3}}
$$

As pointed out above, these positions correspond to minima of $t_{b o s e}(\mathbf{r})$ and $s(\mathbf{r})$. Non-AIM-CPs involve a subtle balance between the local Laplacian of $\rho(\mathbf{r})$, $\nabla^{2} \rho(\mathbf{r}) / \rho(\mathbf{r})$ and a term proportional to the local von Weizsäcker kinetic energy density $8 \tau_{w}(\mathbf{r}) / \rho(\mathbf{r})=(\nabla \rho(\mathbf{r}))^{2} / \rho^{2}(\mathbf{r})$. Moreover, it can be noticed that this will always happen at points of positive Laplacian (see Equation 14). In other words, at closed shell interactions with no AIM CP, i.e. very weak interactions.

The variables in Equation 14 for the non-AIM-CPs are also involved in the oneelectron potential (OEP) [25, 27],

$$
O E P(\mathbf{r})=\frac{1}{4}\left[\frac{\nabla^{2} \rho(\mathbf{r})}{\rho(\mathbf{r})}-\frac{1}{2}\left(\frac{\nabla \rho(\mathbf{r})}{\rho(\mathbf{r})}\right)^{2}\right] .
$$

The regions of negative OEP have been identified as classically allowed regions, in the sense that the kinetic energy density given by -OEP takes positive values. Conversely, the regions where OEP attains positive values, have negative values of this kinetic energy density, and therefore, have been identified as classically forbidden regions. This separation has been used to identify atomic shells and bonding regions as the classically allowed ones. When OEP cancels the ratio between $\nabla^{2} \rho(\mathbf{r}) / \rho(\mathbf{r})$ and $(\nabla \rho(\mathbf{r}))^{2} / \rho^{2}(\mathbf{r})$ is equal to $1 / 2$, whereas it is $4 / 3$ when Equation 14 is satisfied. Thus, any non-AIM CPs of $t_{\text {bose }}(\mathbf{r})$ is anticipated by a zero of OEP, and therefore, by a transition from a classically allowed to a forbidden region.

\subsection{Computational details}

In order to asses the quality of the functions mentioned above as bonding descriptors, we have computed $s(\mathbf{r}), t_{L O L}(\mathbf{r}), t_{b o s e}(\mathbf{r})$ and ELF for several chemically representative systems in their ground states: $\mathrm{N}_{2}$ and $\mathrm{F}_{2}$ as molecules representing apolar covalent and charge-shift bonding type respectively, $\mathrm{H}_{2} \mathrm{O}$ and $\mathrm{CH}_{3} \mathrm{~F}$, as examples of polar covalent bonds, $\mathrm{LiH}$ and $\mathrm{LiF}$ as examples of ionic bonds, and 1,2-ethanediol as an example of NCI. The wavefunctions were calculated at the restricted HartreeFock level with the $631 \mathrm{~g}++\mathrm{dp}$ basis as implemented in GAMESS code [28]. The 1,2-ethanediol geometry has been optimised with the CCSD(T)-F12a/cc-pVDZF12 method as implemented in MOLPRO 2010.1 [29]. All the functions herein presented, were computed with our CHECKIN code, developed explicitly for this work and available upon request. Scalar field visualisation was performed with ParaView version 4.3.1 [30] and VMD version 1.9.1 [31]. 


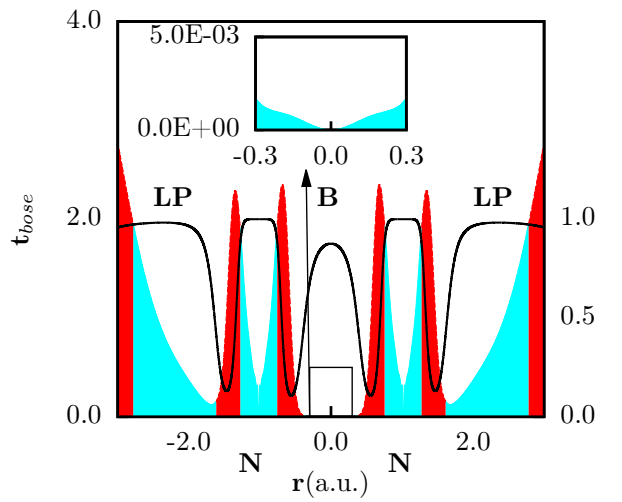

(a)

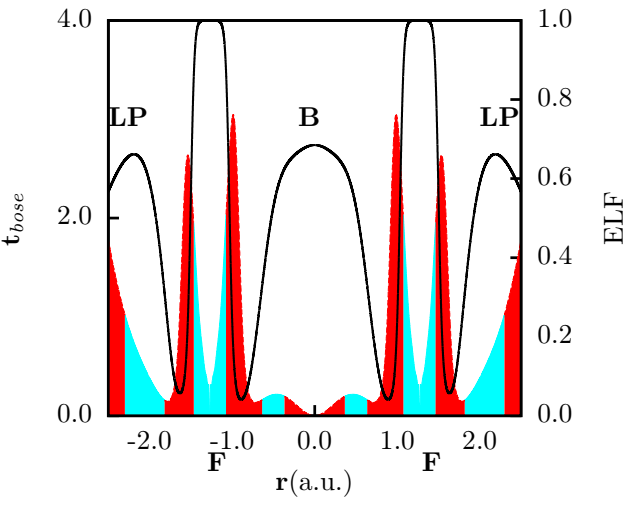

(b)

Figure 2. $t_{\text {bose }}(\mathbf{r})$ along with ELF values (solid black line) for $\mathrm{N}_{2}(\mathrm{a})$ and $\mathrm{F}_{2}(\mathrm{~b})$. Negative (classically allowed) and positive (classically forbidden) regions of $O E P$ are displayed as cyan and red-colored areas respectively. Labels B and LP stand for bond and lone pair ELF basins respectively.

\subsection{Bonding analysis}

\subsubsection{Covalent and ionic interactions}

Figures 2 and 3 display $t_{\text {bose }}(\mathbf{r}), t_{L O L}(\mathbf{r})$ and $s(\mathbf{r})$ along the internuclear axis for $\mathrm{F}_{2}$ and $\mathrm{N}_{2}$. The origin was set at the BCP. Since the equivalence between $s(\mathbf{r})$ and $t_{\text {bose }}(\mathbf{r})$ has been already discussed, for the shake of simplicity we shall only refer to $t_{\text {bose }}(\mathbf{r}) . t_{\text {bose }}(\mathbf{r})$ and $t_{L O L}(\mathbf{r})$ differentiate the core, lone-pairs and interatomic bonding regions as minima separated by maxima. In line with our interpretation of $t_{\text {bose }}(\mathbf{r})$, these minima are regions of electron location, namely regions of electron pair localization as revealed by ELF maxima (See Supporting Information, Figure S2). Nuclear and bond critical points of $\rho(\mathbf{r})$ are identified as positions where $t_{\text {bose }}(\mathbf{r})$ takes a value of zero. Conversely lone pairs are not revealed by critical points of $\rho(\mathbf{r})$, but by critical points of the Laplacian of the electron density. $t_{\text {bose }}(\mathbf{r})$ shows minima at such positions driven by the non-AIM-CP condition (Equation 14), following thereby a transition from a classically forbidden region to a classically allowed region, as may be seen in Figure 2, We highlight that all CPs of $t_{\text {bose }}$ given by Equation 14 are anticipated by roots of OEP, but the opposite is not true; not all roots of OEP are followed by Equation 14. This situation may be found in $\mathrm{N}_{2}$. Even though no CP of $t_{\text {bose }}(\mathbf{r})$ is found between the external core-maxima and the $\mathrm{BCP}$, the former is localised at regions of positive OEP, whereas the latter is found in a region of negative OEP. In contrast, $\mathrm{F}_{2}$ exhibits minima between the $\mathrm{BCP}$ and the external core maxima. We may notice that the $\mathrm{BCP}$ for $\mathrm{F}_{2}$ is localised at a region of positive OEP. It is well known that $\mathrm{F}_{2}$ exhibits a positive value of the Laplacian of the electron density at the BCP, being thereby identified as a region of electron depletion. Because the signs of OEP and $\nabla^{2} \rho(\mathbf{r})$ are the same at CPs of $\rho(\mathbf{r})$, the BCP for $\mathrm{F}_{2}$ is localised at a classically forbidden region.

Whereas the presence of a BCP guarantees the existence of a minimum for $t_{\text {bose }}(\mathbf{r})$ this is not the case for $t_{L O L}(\mathbf{r})$. As a covalent bond indicator it shows a very flat minimum for $\mathrm{CH}_{3} \mathrm{~F}$, and only inflexion points for $\mathrm{H}_{2} \mathrm{O}$ and $\mathrm{LiF}$. As pointed out before the difference between $t_{L O L}(\mathbf{r})$ and $t_{\text {bose }}(\mathbf{r})$ is nothing but the ELF kernel $\chi(\mathbf{r})$. As this difference increases at the BCP, the value of ELF at such point decreases, thus the electron population of the corresponding disynaptic ELF basis decreases until annihilation of the disynaptic basin, as shown in Table 1,

Despite the differences between $t_{L O L}(\mathbf{r})$ and $t_{\text {bose }}(\mathbf{r})$, both functions show for 


\begin{tabular}{lccc}
\hline Molecule & Basin & $\bar{N}(\Omega)$ & $\sigma^{2}$ \\
\hline $\mathrm{N}_{2}$ & $\mathrm{~V}(\mathrm{~N}, \mathrm{~N})$ & 3.24 & 1.39 \\
$\mathrm{~F}_{2}$ & $\mathrm{~V}(\mathrm{~F}, \mathrm{~F})$ & 0.44 & 0.38 \\
$\mathrm{H}_{2} \mathrm{O}$ & $\mathrm{V}(\mathrm{O}, \mathrm{H})$ & 1.71 & 0.78 \\
$\mathrm{CH}_{3} \mathrm{~F}$ & $\mathrm{~V}(\mathrm{C}, \mathrm{F})$ & 0.98 & 0.70 \\
\hline
\end{tabular}

$\mathrm{CH}_{3} \mathrm{~F}$ and $\mathrm{LiF}$ higher core-valence maxima around the less electronegative atom, $\mathrm{C}$ and $\mathrm{Li}$, respectively. The absence of a valence shell in $\mathrm{H}$, avoids this high maxima around the $\mathrm{H}$ atom in the water molecule. It is worthy to say that both functions exhibit a minimum between the BCP and the core-valence maximum of the more electronegative atom for the three polar bonds. This region of electron localization is similar to that found for $\mathrm{F}_{2}$. These results are in line with the ELF analyses, which reveal that $\mathrm{V}(\mathrm{F}, \mathrm{F})$ and $\mathrm{V}(\mathrm{C}, \mathrm{F})$ basins are characterised by a low electron population and relatively high variance. This has been identified as a signature of charge-shift bonding [32, 33].

\subsubsection{Non-covalent interactions}

The ability of $s(\mathbf{r})$ to reveal NCI was firstly discovered by plotting $s(\mathbf{r})$ versus $\rho(\mathbf{r})$. Although pretty new in the chemistry community, the combined analysis of two scalar fields has already been explored in scientific visualisation [34]. This kind of diagrams condensate all the real space information in two variables, and in the particular case of $s(\mathbf{r})$ and $\rho(\mathbf{r})$ they show troughs when $\nabla \rho(\mathbf{r})$ goes to zero. A real space representation of the points in the trough leads to a set of isosurfaces that represents such chemical interactions. This analysis goes beyond local analyses such as AIM, which only focus on the CPs of $\rho(\mathbf{r})$ and not on the surrounding regions. Example of the discrepancies between both the local, as AIM, and regional, as NCI, approaches may be found in 1,2-ethanediol [35]. As shown by Contreras et al. any function of the form $|\nabla \rho| / \rho^{n}$ will reveal NCIs as long as it is able to distinguish them from the exponentially decaying tails of the density [18]. By plotting these functions versus the electron density, they concluded a power of $4 / 3$ used in the reduced density gradient is the best choice, not only for isolating NCI, but also for reducing the range of acceptable values. A power of 1 , used by LED, does not yield such a good resolution as $s(\mathbf{r})$ for non-covalent interactions, but resolves with the same quality both covalent and NCI, since it is bounded by theoretical limits. Although $t_{L O L}(\mathbf{r})$ is not a member of this family of functions, because of the explicit orbital dependence of the positive kinetic energy density, we may analyse its ability to reveal NCI using the same kind of diagram. Although all the points inside the troughs are needed to obtain a real space representation of the chemical interaction, only the lower edge of the curved is needed to identify them. For the sake of simplicity in Figur 4 we splined the lower edges of the $t_{L O L}(\rho), s(\rho)$ and $t_{\text {bose }}(\rho)$ curves.

As shown in Figure 4, LOL is not able to reveal the hydrogen bond in 1,2ethanediol. A representation of these functions along the $\mathrm{O}-\mathrm{H}$ direction confirms this result. Besides the core-valence maxima and the subsequent minimum, $t_{\text {bose }}(\mathbf{r})$ exhibits a minimum corresponding to the weak hydrogen bond, whereas $t_{L O L}(\mathbf{r})$ shows a maximum at this position. Figure 5 displays isosurfaces alongside the maxima and minima of $t_{L O L}(\mathbf{r})$ and $t_{\text {bose }}(\mathbf{r})$. It may be noticed that $t_{\text {bose }}(\mathbf{r})$ reveals a pancake component corresponding to the hydrogen bond, whereas no component is found for $t_{L O L}(\mathbf{r})$ at such position. It is known that LOL [14] and ELF [36] only exhibit 1-saddle $\mathrm{CPs}^{1}$ for non-covalent interactions, following thereby a 2 -saddle $\mathrm{CP}$ of $t_{L O L}(\mathbf{r})$ (Figure 5); a maximum along the $\mathrm{O}-\mathrm{H}$ direction as shown in Figure 4 


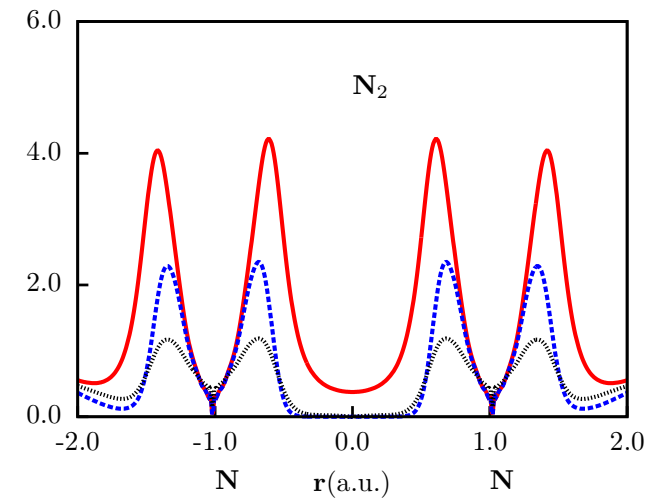

(a)

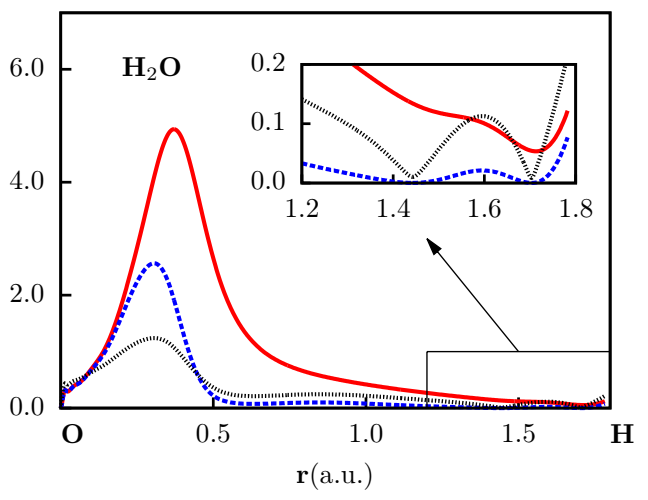

(c)

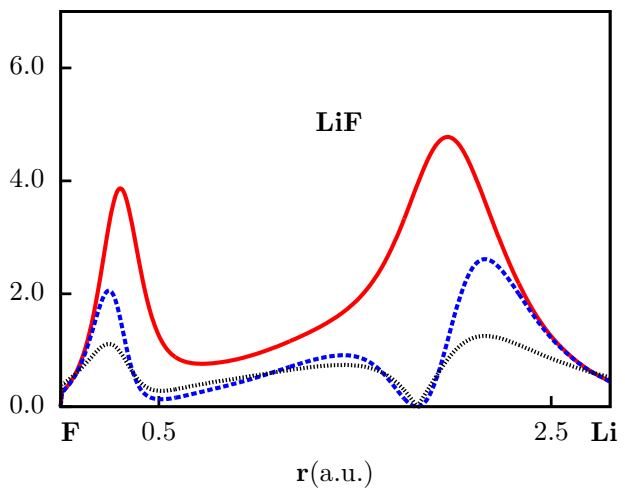

(e)

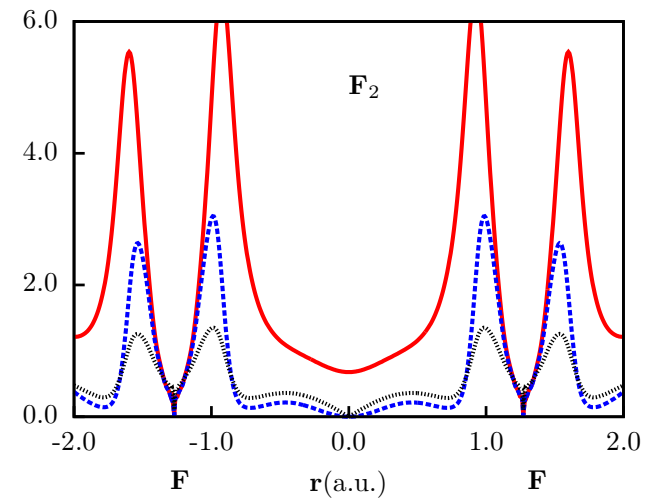

(b)

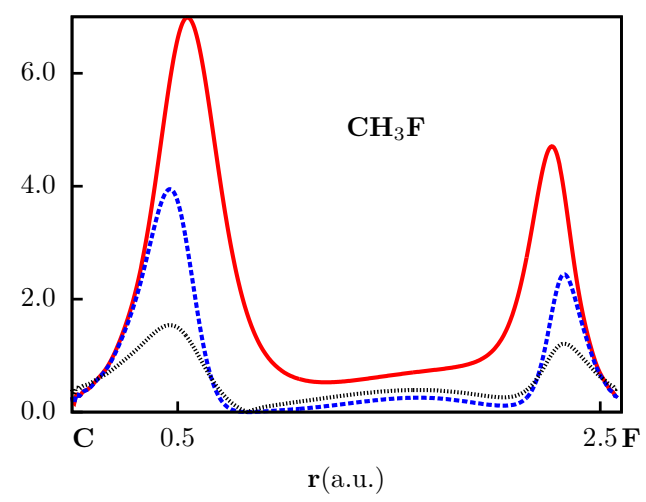

(d)

Figure 3. $t_{L O L}(\mathbf{r})$ (solid red line), $s(\mathbf{r})$ (short dashed black line) and $t_{\text {bose }}$ (dashed blue line) for: (a) $\mathrm{N}_{2}$, (b) $\mathrm{F}_{2}$, (c) $\mathrm{H}_{2} \mathrm{O}$, (d) $\mathrm{CH}_{3} \mathrm{~F}$, and (e) LiF.

and minima in the perpendicular directions. $t_{\text {bose }}(\mathbf{r})$ minimum indicates that the hydrogen bond is driven by a region of electron localisation between the oxygen and the hydrogen atoms. The absence of $t_{L O L}(\mathbf{r})$ minimum prevents ELF from showing any maximum at this position. $t_{\text {bose }}(\mathbf{r})$ and $s(\mathbf{r})$ are therefore, more subtle indicators of electron localisation than $t_{L O L}(\mathbf{r})$, which requires higher degree of electron localisation to exhibit a minimum. 


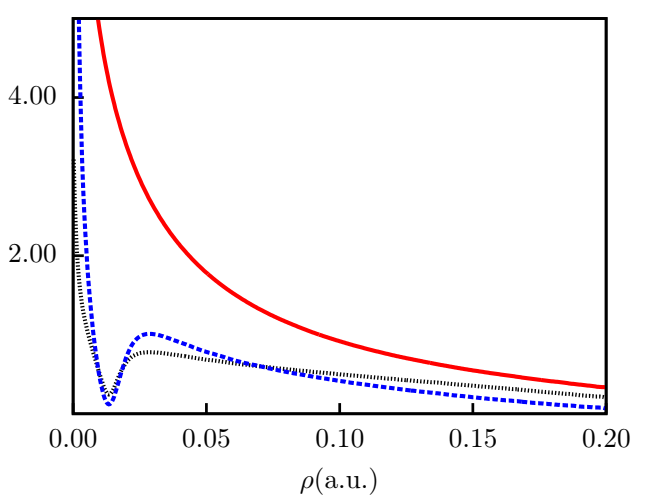

(a)

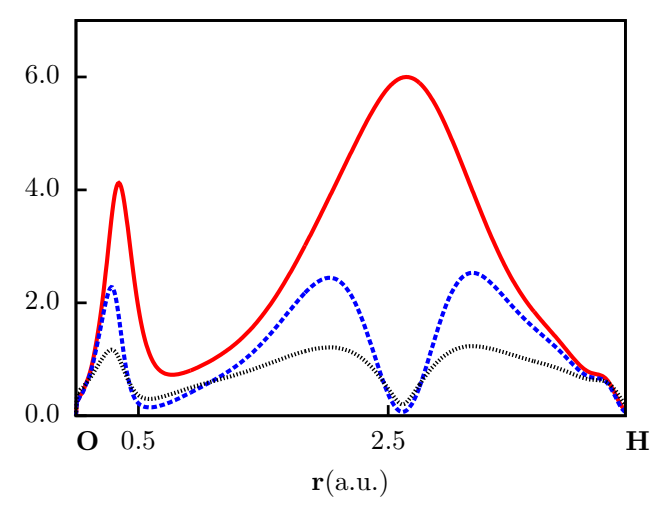

(b)

Figure 4. Hydrogen bonding analysis of the 1,2-ethanediol. $t_{L O L}$ (solid red lines), $s$ (short dashed black line) and $t_{\text {bose }}$ (dashed blue line) in terms of the electron density(a) and the position along the hydrogen bond (b).

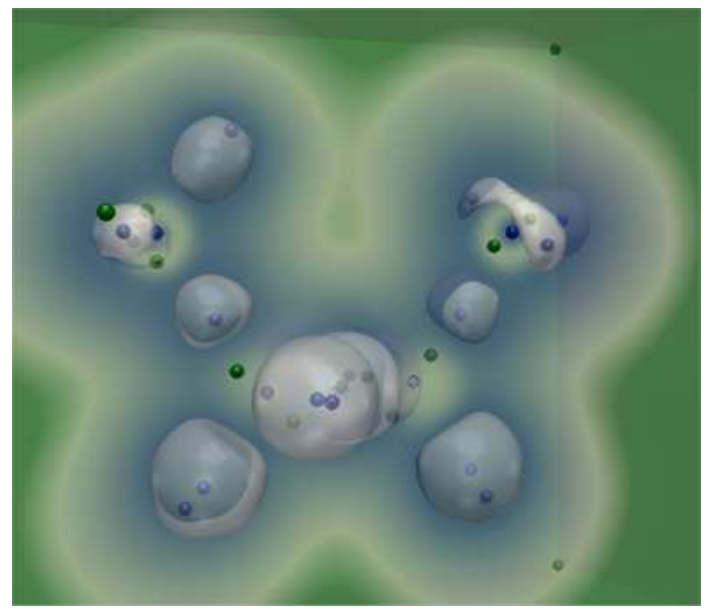

(a)

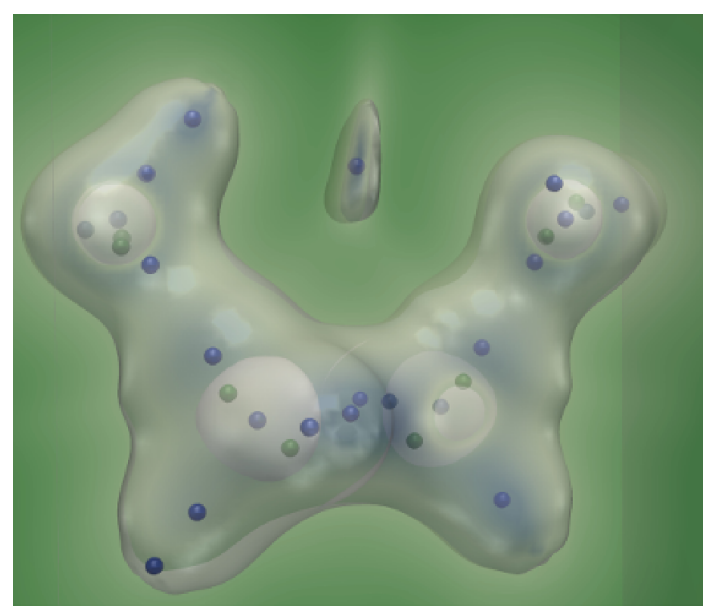

(b)

Figure 5. Critical points of $t_{\text {lol }}(\mathbf{r})(\mathrm{a})$ and $t_{\text {bose }}(\mathbf{r})$ (b) for 1,2-ethanediol. Blue and green spheres represent minima and maxima respectively.

\section{Conclusions}

We have introduced in this contribution the quantity $t_{\text {bose }}(\mathbf{r})$, which is a measure of the bosonic kinetic energy. Covalent, ionic and non-covalent interactions may be easily identified as minima of this function. We have shown that the reduced density gradient $s(\mathbf{r})$ is proportional to the square root of $t_{\text {bose }}(\mathbf{r})$, following thereby the same physical interpretation. The CPs of $t_{\text {bose }}(\mathbf{r})$ are classified in two types; those that match with the CPs of the electron density (AIM-CPs), and those that are precluded by zeros of the one-electron potential (Non-AIM-CPS), and therefore, by transitions from classically allowed to classically forbidden regions. Thus information from $\rho(\mathbf{r})$ and $\nabla^{2} \rho(\mathbf{r})$ is added up.

We have discussed the relationship between $t_{\text {bose }}(\mathbf{r})$ with other descriptors based on the kinetic energy as LOL, ELF and $t_{L O L}(\mathbf{r})$. All of them are able to disclose atomic shells, bonding and lone electron pairs. They reveal NCI as saddle points, thereby being more suited to visualise covalent bonds. We have examined $t_{L O L}(\mathbf{r})$ and $t_{\text {bose }}(\mathbf{r})$ through a set of molecular system representing covalent, ionic and non-covalent interactions. These examples show that $t_{\text {bose }}(\mathbf{r})$ behaves simi- 
larly to $t_{L O L}(\mathbf{r})$ in covalent bonds and additionally, allows visualizing ionic and non-covalent interactions.

\section{Note}

1. The index of a critical point is defined as the number of positive eigenvalues of the hessian matrix at the position of the critical point. In $\mathbb{R}^{3}$, maxima, 1-saddle, 2-saddle and minima critical points have indices $0,1,2$ and 3 respectively.

\section{Acknowledgements}

The authors wish to thank Professor Andreas Savin for his endless strength and discussions. This work was supported partially by the framework of CALSIMLAB under the public grant ANR-11-LABX-0037-01 overseen by the French National Research Agency (ANR) as part of the "Investissements d'Avenir" program (reference: ANR-11-IDEX-0004-02).

\section{References}

[1] E.R. Johnson, S. Keinan, P. Mori-Sanchez, J. Contreras-Garcia, A.J. Cohen and W. Yang, J. Am. Chem. Soc. 132 (18), 6498 (2010).

[2] J.P. Perdew, K. Burke and M. Ernzerhof, Phys. Rev. Lett. 77 (18), 3865 (1996).

[3] V. Sahni, J. Gruenebaum and J. Perdew, Phys. Rev. B 26 (8), 4371 (1982).

[4] E.W. Pearson and R.G. Gordon, J. Chem. Phys. 82 (2), 881 (1985).

[5] V. Tognetti, P. Cortona and C. Adamo, J. Chem. Phys. 128 (3), 034101 (2008).

[6] J. Sun, B. Xiao, Y. Fang, R. Haunschild, P. Hao, A. Ruzsinszky, G.I. Csonka, G.E. Scuseria and J.P. Perdew, Physical review letters 111 (10), 106401 (2013).

[7] A. Zupan, K. Burke, M. Ernzerhof and J.P. Perdew, J. Chem. Phys. 106 (24), 10184 (1997).

[8] A. Zupan, J.P. Perdew, K. Burke and M. Causa, Int. J. Quantum Chem. 61 (5), 835 (1997).

[9] J.M. del Campo, J.L. Gázquez, R.J. Alvarez-Mendez and A. Vela, Int. J. Quantum Chem. 112 (22), 3594 (2012).

[10] K. Finzel, Int. J. Quantum Chem. 114 (22), 1546 (2014).

[11] A.D. Becke and K.E. Edgecombe, J. Chem. Phys. 92, 5397 (1990).

[12] H. Schmider and A. Becke, J. Mol. Struct. 527 (1), 51 (2000).

[13] A.D. Becke and E.R. Johnson, J. Chem. Phys. 124 (22), 221101 (2006).

[14] H. Jacobsen, J. Comput. Chem. 30 (7), 1093 (2009).

[15] H. Jacobsen, Dalton Trans. 39 (23), 5426 (2010).

[16] J. Contreras-García, M. Calatayud, J.P. Piquemal and J. Recio, Comput. Theor. Chem. 998, $193(2012)$.

[17] H.J. Bohorquez, Ph.D. thesis, Dalhousie University 2012.

[18] J. Contreras-García, W. Yang and E.R. Johnson, J. Phys. Chem. A 115 (45), 12983 (2011).

[19] G. Saleh, C. Gatti and L.L. Presti, Comput. Theor. Chem. 1053, 53 (2015).

[20] M. Alonso, T. Woller, F.J. Martín-Martínez, J. Contreras-García, P. Geerlings and F. De Proft, Chem. Eur. J. 20 (17), 4931 (2014).

[21] A. Savin, O. Jepsen, J.r. Flad, O.K. Andersen, H. Preuss and H.G. von Schnering, Angew. Chem. Int. Ed. Engl. 31 (2), 187 (1992).

[22] C. Gatti, Z. Kristallogr. 220 (5/6/2005), 399 (2005).

[23] H.L. Schmider and A.D. Becke, J. Chem. Phys. 116 (8), 3184 (2002).

[24] H.J. Bohórquez and R.J. Boyd, Theor. Chem. Acc. 127 (4), 393 (2010).

[25] G. Hunter, Phys. Rev. Lett. 29 (2), 197 (1986).

[26] A. Savin, J. Mol. Struct. 727 (1), 127 (2005).

[27] R.P. Sagar, A.C. Ku and V.H. Jr, Can. J. Chem. 66 (4), 1005 (1988).

[28] M.W. Schmidt, K.K. Baldridge, J.A. Boatz, S.T. Elbert, M.S. Gordon, J.H. Jensen, S. Koseki, N. Matsunaga, K.A. Nguyen, S. Su et al., J. Comput. Chem. 14 (11), 1347 (1993). 
[29] H.J. Werner, P.J. Knowles, G. Knizia, F.R. Manby, M. Schütz, P. Celani, T. Korona, R. Lindh, A. Mitrushenkov, G. Rauhut, K.R. Shamasundar, T.B. Adler, R.D. Amos, A. Bernhardsson, A. Berning, D.L. Cooper, M.J.O. Deegan, A.J. Dobbyn, F. Eckert, E. Goll, C. Hampel, A. Hesselmann, G. Hetzer, T. Hrenar, G. Jansen, C. Köppl, Y. Liu, A.W. Lloyd, R.A. Mata, A.J. May, S.J. McNicholas, W. Meyer, M.E. Mura, A. Nicklass, D.P. O’Neill, P. Palmieri, D. Peng, K. Pflüger, R. Pitzer, M. Reiher, T. Shiozaki, H. Stoll, A.J. Stone, R. Tarroni, T. Thorsteinsson and M. Wang, MOLPRO, version 2012.1, a package of ab initio programs Unirversity College Cardiff Consultants Limited: Cardiff, U. K.

[30] J. Ahrens, B. Geverci and L. Charles, ParaView: An End-User Tool for Large Data Visualization, Visualization Handbook Elservier 2005.

[31] W. Humphrey, A. Dalke and K. Schulten, J. Mol. Graphics 14, 33 (1996).

[32] R. Llusar, A. Beltrán, J. Andrés, S. Noury and B. Silvi, J. Comput. Chem. 20 (14), 1517 (1999).

[33] S. Shaik, D. Danovich, B. Silvi, D.L. Lauvergnat and P.C. Hiberty, Chem. Eur. J. 11 (21), $6358(2005)$

[34] H. Carr, Z. Geng, J. Tierny, A. Chattopadhyay and A. Knoll, Comput. Graph. Forum 34 (3), $241(2015)$.

[35] J.R. Lane, J. Contreras-García, J.P. Piquemal, B.J. Miller and H.G. Kjaergaard, J. Chem. Theory. Comput. 9 (8), 3263 (2013).

[36] M. Alikhani, F. Fuster and B. Silvi, Struct. Chem. 16 (3), 203 (2005). 